\title{
Interaction pattern in a language classroom amongst special needs children: Imaginative learning approach
}

\begin{abstract}
This research investigates the interaction pattern amongst special needs children while learning the second language. These children have asperger syndrome or asperger's disorder which is a neurobiological disorder that described a pattern of behaviors in young boys or girls who had normal intelligence and language development, but who also exhibited autisticlike behaviors and marked deficiencies in social and communication skills (Kirby, 2005). Children with asperger syndrome can exhibit a variety of characteristics and the disorder can range from mild to severe. Children with asperger syndrome show marked deficiencies in social skills have difficulties with transitions or changes and prefer sameness. Therefore, this paper focuses on the nature, number and balance of interactions of ten subjects with asperger syndrome and further investigates the interaction pattern and reactions which occurred while using the Imaginative Learning Approach (IMA) (Normaliza Abd Rahim, 2006) in learning the second language. IMA is an activity where the subjects were given a scenario, for e.g. a hospital. A corner will be set up like a hospital and the subjects were given three times a week to play with their peers at the corner. The nature, number and balance of interactions were video-taped to find out the interaction pattern. The subjects consist of five males and five females ( 7 to 15 years old) with asperger syndrome from schools in Selangor, Malaysia. The introduction of IMA to the subjects managed to enhance the learning of the second language. It is found that asperger learners, able to concentrate and interact with their peers in IMA.
\end{abstract}

Keyword: Imaginative learning approach, special needs children 